\title{
CLOSURE THEOREMS FOR ORIENTOR FIELDS ${ }^{1}$
}

\author{
BY LAMBERTO CESARI \\ Communicated by M. H. Protter, November 20, 1972
}

1. Introduction. In the theory of optimization a number of closure and lower closure theorems have been obtained in different contexts and under a variety of conditions and modes of convergence. Particularly, "seminormality" conditions have been playing different roles.

In $\S 3$ of the present paper we show that much more general and more satisfactory results can be obtained in terms of orientor fields. The closure and lower closure theorems so obtained are proved indeed under a minimum of conditions, and they contain as particular cases analogous theorems so far obtained in the more particular context of Mayer and Lagrange problems (§4). The proofs of the present theorems are given in [1] and are rather simple. Also, the statements show the interplay of modes of convergence versus seminormality conditions. The results are based on the use of the Banach-Saks-Mazur theorem on weak convergence in normed spaces.

In $\$ 4$ we state closure and lower closure theorems for Mayer and Lagrange problems. In particular, theorems of this type are stated under no seminormality condition at all, but under suitable Lipschitz-type, and, more satisfactorily, under sole growth-type conditions [3]. Finally, even these Lipschitz-type and growth-type conditions can be dispensed with. Indeed, on the basis of the present considerations, we prove in [2] lower semicontinuity theorems for free problems of the calculus of variations in which only continuity and convexity hypotheses are made. Corresponding closure and lower closure theorems for orientor fields, Mayer and Lagrange problems are also proved in [2].

2. Notations. Points in spaces $E_{v}, E_{n}, E_{r+1}$ will be denoted by $t=\left(t^{1}, \ldots, t^{v}\right), x=\left(x^{1}, \ldots, x^{n}\right)$, and $\left(z^{0}, z\right)=\left(z^{0}, z^{1}, \ldots, z^{r}\right)$, or $(\eta, \xi)=$ $\left(\eta, \xi^{1}, \ldots, \xi^{r}\right)$. Let $A$ be a given subset of $E_{v} \times E_{n}, A_{0}$ the projection of $A$ on $E_{v}$, and $A(t)=\left[x \in E_{n} \mid(t, x) \in A\right], t \in A_{0}$. We denote by $\operatorname{cl} Z$, co $Z$, $|Z|$, the closure, the convex hull, and the outer Lebesgue measure of a set $Z$ in $E_{N}$. For every $(t, x) \in A$ a subset $R(t, x)$ of $E_{r+1}$ is assigned. Let $G, T$ be given subsets of $A_{0}, G$ measurable with finite measure, $T$ of measure zero, $T \subset G \subset E_{v}$. We denote by $A_{G}$ and $A_{T}$ the sets $A_{G}=[(t, x) \in A \mid t \in G]$,

AMS (MOS) subject classifications (1970). Primary 49A50; Secondary 49A10, 49A20.

${ }^{1}$ This research was partially supported by US-AFOSR Research Project 71-2122 at the University of Michigan. 
$A_{T}=[(t, x) \in A \mid t \in T]$. For every $(\bar{t}, \bar{x}) \in A$ and $\delta>0$ we denote by $N_{\delta ; \bar{z}}(\bar{x})$ the set of all points $(\bar{z}, x) \in A$ at a distance $\leqq \delta$ from $(\bar{t}, \bar{x})$. Only the following concepts of upper semicontinuity of variable sets are of interest here. We say that the sets $R(t, x)$ satisfy (Kuratowski's) property $(\mathrm{K})$ with respect to $x$ at $(\bar{t}, \bar{x})$ provided

$$
R(\bar{t}, \bar{x})=\bigcap_{\delta>0} \mathrm{cl} \bigcup_{(\bar{t}, x) \in N_{\delta ; \bar{t}}(\bar{x})} R(\bar{t}, x) .
$$

We say that the same sets $R(t, x)$ satisfy property (Q) with respect to $x$ at $(\bar{t}, \bar{x})$ provided

$$
R(\bar{t}, \bar{x})=\bigcap_{\delta>0} \operatorname{clco} \bigcup_{(\bar{t}, x) \in N_{\delta ;} \bar{t}(\bar{x})} R(\bar{t}, x) .
$$

We shall say that the sets $R(t, x)$ satisfy property $(\mathrm{K})$, or property $(\mathrm{Q})$ with respect to $x$ in a subset $A^{\prime}$ of $A$ if the corresponding property holds at every $(\bar{t}, \bar{x}) \in A^{\prime}$. Sets $R(t, x)$ possessing property $(\mathrm{K})$ are closed, and sets possessing property $(\mathrm{Q})$ are closed and convex, as intersections of sets having the same properties. We proved elsewhere that property (Q) for Lagrange problems is the extension of Tonelli's and McShane's seminormality condition for free problems. We shall consider systems of measurable functions $\xi(t), \eta(t), x(t), t \in G$, satisfying the orientor field relations

$$
x(t) \in A(t), \quad(\eta(t), \xi(t)) \in R(t, x(t)), \quad t \in G \quad \text { (a.e.). }
$$

In applications, the sets $R(t, x)$ have a particular structure, namely they have the property $(\pi)$ : for every $\left(\bar{z}^{0}, z\right) \in R(t, x)$ and $z^{0} \geqq \bar{z}^{0}$, then also $\left(z^{0}, z\right) \in R(t, x)$.

3. The main statements: the lower closure theorems for orientor fields.

(3.i) If $T \subset G \subset E_{v},|T|=0,|G|<\infty$, if the sets $A(t)$ are closed for $t \in G-T$, if the sets $R(t, x)$ satisfy property (Q) with respect to $x$ in $A_{G}-A_{T}$, if $\xi(t), x(t), \xi_{k}(t), \eta_{k}(t), x_{k}(t), \lambda(t), \lambda_{k}(t), t \in G, k=1,2, \ldots$, are measurable functions, $\xi, \xi_{k} \in\left(L_{1}(G)\right)^{r}, \eta_{k} \in L_{1}(G)$, such that

$$
\begin{gathered}
x_{k}(t) \in A(t), \quad\left(\eta_{k}(t), \xi_{k}(t)\right) \in R\left(t, x_{k}(t)\right), \quad t \in G \quad(\text { a.e. }), k=1,2, \ldots, \\
-\infty<i=\liminf _{k \rightarrow \infty} \int_{G} \eta_{k}(t) d t<\infty
\end{gathered}
$$

$$
\begin{gathered}
\xi_{k} \rightarrow \xi \text { weakly in }\left(L_{1}(G)\right)^{r}, x_{k}(t) \rightarrow x(t) \text { in measure in } G \text { as } k \rightarrow \infty, \\
\eta_{k}(t) \geqq \lambda_{k}(t), \quad \lambda, \lambda_{k} \in L_{1}(G), \quad \lambda_{k} \rightarrow \lambda \text { weakly in } L_{1}(G),
\end{gathered}
$$

then there is a function $\eta(t), t \in G, \eta \in L_{1}(G)$, such that

$$
\begin{gathered}
x(t) \in A(t), \quad(\eta(t), \xi(t)) \in R(t, x(t)), \quad t \in G \quad \text { (a.e.) } \\
\int_{G} \eta(t) d t \leqq i .
\end{gathered}
$$


In this formulation of statement (3.i) the inequality $i>-\infty$ is a consequence of the requirements on the functions $\lambda, \lambda_{k}$. Functions $\lambda, \lambda_{k}$ satisfying these requirements are easily found in a number of situations, for instance if one of the following conditions is satisfied:

$(\alpha)$ There are a function $\psi(t) \geqq 0, t \in G, \psi \in L_{1}(G)$, and a constant $\gamma \geqq 0$ such that $\left(z^{0}, z\right) \in R(t, x)$ implies $z^{0} \geqq-\psi(t)-\gamma|z|$.

$(\beta) x, x_{k} \in\left(L_{p}(G)\right)^{n},\left\|x_{k}-x\right\|_{p} \rightarrow 0,1 \leqq p<\infty$, and there are $\psi$ as above, and constants $\gamma, \gamma^{\prime} \geqq 0$ such that $\left(z^{0}, z\right) \in R(t, x)$ implies $z^{0} \geqq-\psi(t)-$ $\gamma|x|^{p}-\gamma^{\prime}|z|$.

Other analogous conditions are stated in [1]. Examples in [1] show that the conclusions of (3.i) may not hold if the conditions relative to the functions $\lambda, \lambda_{k}$ are not satisfied. However, statements analogous to (3.i) have been proved under alternate hypotheses concerning the functions $\lambda, \lambda_{k}$.

For instance, for the functions $\lambda, \lambda_{k}$ we may require only that they are measurable $(\lambda$ can even take values $-\infty)$, and that they satisfy the alternate requirements :

$$
\begin{aligned}
& \eta_{k}(t) \geqq \lambda_{k}(t), \quad \lambda_{k}(t) \geqq \lambda_{k+1}(t), \quad \lambda_{k}(t) \rightarrow \lambda(t) \quad \text { pointwise, } \\
& \left(\lambda_{k}(t), \xi_{k}(t)\right) \in R\left(t, x_{k}(t)\right), \quad t \in G \quad(\text { a.e. }), k=1,2, \ldots .
\end{aligned}
$$

In this situation, however, the function $\eta$ in statement (5.i) may be only measurable, finite almost everywhere, with $L$-integral $\int_{G} \eta(t) d t$ finite, or $-\infty$, satisfying (2) as stated. If in addition the sets $R(t, x)$ have property $(\pi)$, then $\eta \in L_{1}(G)$.

(3.ii) The same as (3.i) where the sets $R(t, x)$ satisfy property $(\mathrm{K})$ with respect to $x$ in $A_{G}-A_{T}$, and $\xi_{k}(t) \rightarrow \xi(t)$ in measure in $G$ as $k \rightarrow \infty$.

There are situations where the sequences $\xi_{k}(t), \eta_{k}(t), x_{k}(t), t \in G, k=$ $1,2, \ldots$, satisfying $\left(\eta_{k}(t), \xi_{k}(t)\right) \in R\left(t, x_{k}(t)\right), t \in G$ (a.e.), with $\xi_{k} \rightarrow \xi$ weakly in $\left(L_{1}(G)\right)^{r}, \lim \int_{G} \eta_{k}(t) d t=i, x_{k}(t) \rightarrow x(t)$ in measure in $G$ as $k \rightarrow \infty$, can be replaced by sequences $\bar{\xi}_{k}(t), \bar{\eta}_{k}(t), x(t), t \in G, k=1,2, \ldots$, satisfying $\left(\bar{\xi}_{k}(t), \bar{\eta}_{k}(t)\right) \in R(t, x(t)), t \in G$, and where $\bar{\xi}_{k}$ still converges to $\xi$ weakly in $\left(L_{1}(G)\right)^{r}$ and $\lim \int_{G} \bar{\eta}_{k}(t) d t=i$. Actually, it occurs that $\delta_{k}(t)=\xi_{k}(t)-$ $\bar{\xi}_{k}(t) \rightarrow 0$ weakly in $\left(L_{1}(G)\right)^{r}$ and $\delta_{k}^{0}(t)=\eta_{k}(t)-\bar{\eta}_{k}(t) \rightarrow 0$ weakly in $L_{1}(G)$ as $k \rightarrow \infty$. There is a large class of problems for which this can be proved, and the sets $R(t, x)$ need be assumed only convex and closed (no property $(\mathrm{Q})$ or $(\mathrm{K})$ required). The following simple statement suffices for these problems.

(3.iii) The same as (3.i) where now the sets $R(t, x)$ are only convex and closed for every $(t, x) \in A_{G}-A_{T}$, where $\xi(t), x(t), \xi_{k}(t), \eta_{k}(t), t \in G, k=$ $1,2, \ldots$, are measurable functions, $\xi, \xi_{k} \in\left(L_{1}(G)\right)^{r}, \eta_{k} \in L_{1}(G)$, such that $x(t) \in A(t), \quad\left(\eta_{k}(t), \quad \xi_{k}(t)\right) \in R(t, x(t)), t \in G$ (a.e.), $k=1,2, \ldots,-\infty<i=$ $\lim \inf \int_{G} \eta_{k}(t) d t<+\infty$, and $\xi_{k} \rightarrow \xi$ weakly in $\left(L_{1}(G)\right)^{r}$. Then, there is a 
function $\eta(t), t \in G, \eta \in L_{1}(G)$, such that $(\eta(t), \xi(t)) \in R(t, x(t)), t \in G$ (a.e.), and $\int_{G} \eta(t) d t \leqq i$.

These theorems (3.i-iii) apply to Lagrange problems (see $\$ 4$ below) as well as to Mayer problems with comparison functionals. For Mayer problems without comparison functionals one has only to take $R(t, x) \subset$ $\left[\left(z^{0}, z\right) \in E_{r+1} \mid z^{0}=0\right]$ and $\eta(t)=\eta_{k}(t)=0$.

Theorems analogous to the ones above hold where $\eta$ is a vector function of some $s$ components, and the sets $R(t, x)$ are subsets of $E_{r+s}$.

4. Lagrange and Mayer problems. Points in an auxiliary space $E_{m}$ will be denoted by $u=\left(u^{1}, \ldots, u^{m}\right)$. The sets $A, A_{0}, A(t), G, T$ are as in $\S 2$, and now for every $(t, x) \in A$ a subset $U(t, x)$ of $E_{m}$ is assigned. Let $M$ denote the set $M=[(t, x, u) \mid(t, x) \in A, u \in U(t, x)]$, let

$$
f_{0}(t, x, u), f(t, x, u)=\left(f_{1}, \ldots, f_{r}\right)
$$

be given functions defined on $M$, and let $R(t, x), Q(t, x)$ denote the sets

$$
\begin{aligned}
& R(t, x)=\left[\left(z^{0}, z\right) \mid z^{0} \geqq f_{0}(t, x, u), z=f(t, x, u), u \in U(t, x)\right] \subset E_{r+1}, \\
& Q(t, x)=[z \mid z=f(t, x, u), u \in U(t, x)] \subset E_{r} .
\end{aligned}
$$

We say that a Carathéodory continuity condition $(\mathrm{C})$ is satisfied provided for every $\varepsilon>0$ there is some compact subset $K$ of $G$ such that $|G-K|<\varepsilon$, the sets $A_{K}=[(t, x) \in A \mid t \in K], M_{K}=[(t, x, u) \in M \mid t \in K]$ are closed, and $f_{0}(t, x, u), f(t, x, u)$ are continuous on $M_{K}$. Then, the sets $A(t)$ are certainly closed for almost all $t \in G$. The following statements are corollaries of (3.i-iii).

(4.i) If $T \subset G \subset E_{v},|T|=0,|G|<\infty$, if condition (C) holds, if the sets $R(t, x)$ defined above satisfy property $(\mathrm{Q})$ with respect to $x$ in $A_{G}-A_{T}$, if $\xi(t), x(t), \xi_{k}(t), \eta_{k}(t), x_{k}(t), u_{k}(t), \lambda(t), \lambda_{k}(t), t \in G, k=1,2, \ldots$, are measurable functions, $\xi, \xi_{k} \in\left(L_{1}(G)\right)^{r}, \eta_{k}, \lambda, \lambda_{k} \in L_{1}(G)$, such that

$$
\begin{aligned}
& x_{k}(t) \in A(t), \quad u_{k}(t) \in U\left(t, x_{k}(t)\right), \quad \xi_{k}(t)=f\left(t, x_{k}(t), u_{k}(t)\right), \\
& \eta_{k}(t)=f_{0}\left(t, x_{k}(t), u_{k}(t)\right), \quad t \in G \quad \text { (a.e.), } k=1,2, \ldots, \\
& -\infty<i=\liminf \int_{G} \eta_{k}(t) d t<+\infty, \\
& \xi_{k} \rightarrow \xi \text { weakly in }\left(L_{1}(G)\right)^{r}, \quad x_{k}(t) \rightarrow x(t) \text { in measure, } \\
& \eta_{k}(t) \geqq \lambda_{k}(t), \quad \lambda_{k} \rightarrow \lambda \quad \text { weakly in } L_{1}(G),
\end{aligned}
$$

then there is a measurable function $u(t), t \in G$, such that

$$
\begin{gathered}
x(t) \in A(t), \quad u(t) \in U(t, x(t)), \\
\xi(t)=f(t, x(t), u(t)), \quad t \in G \quad \text { (a.e.), }
\end{gathered}
$$


and such that, if $\eta_{0}(t)=f_{0}(t, x(t), u(t)), t \in G$, then $\eta_{0}$ is measurable and

$$
\int_{G} \eta_{0}(t) d t \leqq i .
$$

Here $\int_{G} \eta_{0}(t) d t$ as a Lebesgue integral exists (finite, or $-\infty$ ) and (5) holds as stated. Indeed, if $\eta(t), t \in G$, is the function whose existence was proved in (3.i), we have here $\eta_{0}(t)=f_{0}(t, x(t), u(t)) \leqq \eta(t), t \in G$ (a.e.). Under the sole hypotheses of (4.i) the integral $\int_{G} \eta_{0}(t) d t$ may well be $-\infty$, as examples show. Nevertheless, there is a variety of conditions, usually satisfied in applications, which guarantee that $\eta_{0}$ is of class $L_{1}(G)$, the integral $\int_{G} \eta_{0}(t) d t$ is finite, (5) holds as stated, and the conditions relative to the functions $\lambda, \lambda_{k}$ are all satisfied. Here are some of these conditions.

$\left(\alpha_{0}\right) f_{0}(t, x, u) \geqq-\psi(t)-\gamma|f(t, x, u)|$ for all $(t, x, u) \in M$, for some given function $\psi(t) \geqq 0, t \in G, \psi \in L_{1}(G)$, and constant $\gamma$.

$\left(\beta_{0}\right) f_{0}(t, x, u) \geqq-\psi(t)-\gamma|x|^{p}-\gamma^{\prime}|f(t, x, u)|$ for all $(t, x, u) \in M$, for some $\psi$ as above and constants $\gamma, \gamma^{\prime} \geqq 0$, and in addition we know that in (4.i) we have $x, x_{k} \in\left(L_{p}(G)\right)^{r},\left\|x_{k}-x\right\|_{p} \rightarrow 0$ as $k \rightarrow \infty, 1 \leqq p<\infty$.

Analogous conditions can be formulated for functions $x, x_{k}$, or $u, u_{k}$ in suitable classes $L_{p}$ or $L_{\infty}$.

(4.ii) The same as (4.i) where the sets $R(t, x)$ satisfy only property (K) with respect to $x$ in $A_{G}-A_{T}$ and $\xi_{k} \rightarrow \xi$ in measure in $G$ as $k \rightarrow \infty$.

Finally, if the sets $U(t)$ depend on $t$ only, so that the differences

$$
\begin{aligned}
& \delta_{k}(t)=\xi_{k}(t)-\bar{\xi}_{k}(t)=f\left(t, x_{k}(t), u_{k}(t)\right)-f\left(t, x(t), u_{k}(t)\right), \\
& \delta_{k}^{0}(t)=\eta_{k}(t)-\bar{\eta}_{k}(t)=f_{0}\left(t, x_{k}(t), u_{k}(t)\right)-f_{0}\left(t, x(t), u_{k}(t)\right),
\end{aligned}
$$

$$
t \in G, k=1,2, \ldots
$$

exist, and we know that $\delta_{k}(t) \rightarrow 0, \delta_{k}^{0} \rightarrow 0$ weakly in $L_{1}$, then we can replace the sequence $\xi_{k}(t), \eta_{k}(t), x_{k}(t), u_{k}(t), t \in G, k=1,2, \ldots$, by the sequence $\bar{\xi}_{k}(t), \bar{\eta}_{k}(t), x(t), u_{k}(t), t \in G, k=1,2, \ldots$, satisfying now the orientor field relation $\left(\bar{\xi}_{k}(t), \bar{\eta}_{k}(t)\right) \in R(t, x(t)), t \in G$ (a.e.), $k=1,2, \ldots$. The following theorem is now a corollary of (3.iii).

(4.iii) The same as (4.i), where now the sets $R(t, x)$ are only convex and closed for every $(t, x) \in A_{G}-A_{T}$, the sets $U(t)$ depend only on $t$, relations (3) hold, with $\xi, \xi_{k}, \delta_{k} \in\left(L_{1}(G)\right)^{r}, \eta_{k}, \delta_{k}^{0} \in L_{1}(G), \xi_{k} \rightarrow \xi, \delta_{k} \rightarrow 0$ weakly in $\left(L_{1}(G)\right)^{r}, \delta_{k}^{0} \rightarrow 0$ weakly in $L_{1}(G)$. Then, there is a measurable function $u(t), t \in G$, such that (4) and (5) hold.

Theorems (4.i-iii) apply to abstract multidimensional Lagrange problems, and to Mayer problems with comparison functional. Analogous theorems for Mayer problems without comparison functional (closure theorems) are simply obtained by taking $f_{0}=0, \eta(t)=\eta_{k}(t)=0$. 
5. A few criteria for $\delta_{k}, \delta_{k}^{0}$ to approach zero in $L_{1}$. We list here a few conditions on $f$ and $f_{0}$ under which $\left\|\delta_{k}\right\|_{1},\left\|\delta_{k}^{0}\right\|_{1} \rightarrow 0$. For the sake of brevity we limit ourselves to $f$ and the differences $\delta_{k}$. More conditions and details are discussed in [3]. These conditions are of the Lipschitz-type $\left(F_{p}, F_{\infty}\right.$ below), and of the growth-type $\left(G_{p q}, G_{\infty q}, H_{q}\right.$, etc.). Also these results, that we mention here briefly for their practical significance and simple proof, improve on previous known ones. However, it is worth mentioning here that, as we prove in [4], the same conditions under consideration imply some weak form of property $(\mathrm{Q})$, which is still sufficient for the application of our main argument in (3.i) and (4.i).

$\left(F_{p}\right)$ For $1 \leqq p<\infty, x, x_{k} \in\left(L_{p}(G)\right)^{n},\left\|x_{k}-x\right\|_{p} \rightarrow 0$, and

$$
\left|f\left(t, x_{k}(t), u_{k}(t)\right)-f\left(t, x(t), u_{k}(t)\right)\right| \leqq F_{k}(t) h\left(\left|x_{k}(t)-x(t)\right|\right), \quad t \in G,
$$

$k=1,2, \ldots$, where $h(\zeta), 0 \leqq \zeta<+\infty$, is a given monotone nondecreasing function with $h(0+)=0, h(\zeta) \leqq c \zeta^{\gamma}, c \geqq 0,0<\gamma \leqq p$, for all $\zeta \geqq \zeta_{0} \geqq 0$ $\left(c, \gamma, \zeta_{0}\right.$ given constants), and $F_{k}(t) \geqq 0, t \in G, F_{k} \in L_{p^{\prime}}(G)$, given functions with $p^{\prime}=p /(p-\gamma),\left(p^{\prime}=\infty\right.$ if $\left.\gamma=p\right)$, and $\left\|F_{k}\right\|_{p^{\prime}} \leqq C$, a given constant.

$\left(G_{p q}\right)$ For $1 \leqq p, q<\infty, x, x_{k} \in\left(L_{p}(G)\right)^{n}, u, u_{k} \in\left(L_{q}(G)\right)^{m},\|x\|_{p},\left\|x_{k}\right\|_{p} \leqq$ $L_{0},\|u\|_{q},\left\|u_{k}\right\|_{q} \leqq L\left(L_{0}, L\right.$ given constants), $x_{k}(t) \rightarrow x(t)$ in measure in $G$ as $k \rightarrow \infty$, and there are constants $c, c^{\prime}, \alpha, \beta, 0<\alpha \leqq p, 0<\beta \leqq q$, and a function $\psi(t) \geqq 0, t \in G, \psi \in L_{1}(G)$, such that for all $(t, x, u),(t, y, u) \in M$ we have

$$
|f(t, x, u)-f(t, y, u)| \leqq \psi(t)+c\left(|x|^{p-\alpha}+|y|^{p-\alpha}\right)+c^{\prime}|u|^{q-\beta} .
$$

$\left(H_{q}\right)$ For $1 \leqq q<\infty, x, x_{k}$ measurable, $x_{k}(t) \rightarrow x(t)$ in measure in $G$ as $k \rightarrow \infty, u, u_{k} \in\left(L_{q}(G)\right)^{m},\|u\|_{q},\left\|u_{k}\right\|_{q} \leqq L$, a constant, and there are other constants $c^{\prime}, \beta, 0<\beta \leqq q$, and a function $\psi$ as above such that for all $(t, x, u),(t, y, u) \in M$ we have

$$
|f(t, x, u)-f(t, y, u)| \leqq \psi(t)+c^{\prime}|u|^{q-\beta} .
$$

Conditions $\left(G_{\infty q}\right),\left(H_{\infty}\right)$, and others can be formulated analogously.

\section{REFERENCES}

1. L. Cesari, Closure theorems for orientor fields and weak convergence, Arch. Rational Mech. Anal. (to appear).

2. ditions, Ann. Mat. Pura Appl. (to appear).

3. L. Cesari and M. B. Suryanarayana, Closure theorems without seminormality conditions, J. Optimization Theory Appl. (to appear).

4. - Convexity and property $(\mathrm{Q})$ in optimal control theory, SIAM J. Control (to appear).

Department of Mathematics, University of Michigan, Ann Arbor, Michigan 48104 